\title{
Gene expression changes in human mesenchymal stem cells from patients with osteoporosis
}

\author{
LIANYONG LIU $^{1 *}$, QINGYUN ZHU ${ }^{2 *}$, JINGNAN WANG $^{1}$, QIAN XI ${ }^{1}$, HONGLING ZHU ${ }^{1}$ and MINGJUN GU ${ }^{1}$ \\ Departments of ${ }^{1}$ Endocrinology and ${ }^{2}$ Gastroenterology, Shanghai Pudong Gongli Hospital, Shanghai 200135, P.R. China
}

Received February 25, 2014; Accepted December 9, 2014

DOI: $10.3892 / \mathrm{mmr} .2015 .3514$

\begin{abstract}
The aim of the present study was to investigate the underlying molecular mechanisms of osteoporosis and to identify novel candidate genes involved in this disease. The gene expression profile of GSE35958 was downloaded from Gene Expression Omnibus, including five samples of human mesenchymal stem cells from patients with osteoporosis and four control samples. Differentially expressed genes (DEGs) were initially identified following an analysis using Student's t-test. Subsequently, a protein-protein interaction (PPI) network of the significant pathways was constructed, based on the Human Protein Reference Database. In the significant pathways, DEGs were screened using cut-off criteria of FDR $<0.1$ and $\mid \log _{2} \mathrm{FCl}>1.5$. A co-change network for pathways was also constructed using the method of cumulative hypergeometric probability distribution. Finally, the transcriptional regulatory network for DEGs was constructed based on the TRANSFAC database. In total, 1,127 DEGs, including 554 upregulated and 573 downregulated DEGs, were screened. The constructed PPI network for the DEGs involved in the two significant pathways, including focal adhesion and lysosome, demonstrated that the five DEGs with a high degree $(>60)$ were $\beta$-catenin, SHC-transforming protein $1, \mathrm{RAC}-\alpha$ serine/threonine-protein kinase, caveolin 1 and filamin A, with degrees of 135, 117, 117, 73 and 63, respectively. The pathway with the degree of 22 in the constructed co-change network was neuroactive ligand receptor interaction. The nine genes with a high $(\geq 9)$ degree in the constructed transcriptional regulatory network were REL-associated protein, upstream stimulatory factor 1 , specificity protein 1, Fos-related antigen 1, cyclin-dependent kinase inhibitor 1A, upstream stimulatory factor 2, ETS domain-containing protein Elk-1, JUND and retinoic acid
\end{abstract}

Correspondence to: Dr Mingjun Gu, Department of Endocrinology, Shanghai Pudong Gongli Hospital, 219 Miao Pu Road, Shanghai 200135, P.R. China

E-mail: gmingjun@hotmail.com

${ }^{*}$ Contributed equally

Key words: osteoporosis, pathway analysis, transcriptional regulatory network receptor $\alpha$, with degrees of $29,27,19,18,17,13,11,11$ and 9, respectively. The DEGs with high degree in the PPI and transcriptional regulatory networks may be candidate target molecules, which may be used to monitor, diagnose and treat osteoporosis.

\section{Introduction}

Osteoporosis, characterized by the loss of bone mass and strength, and the development of microarchitecture impairment leading to fragility fractures, has become a significant clinical problem in health care services dealing with aging populations $(1,2)$. The susceptibility to osteoporosis is regulated by a variety of factors, such as genetic variants, age, sex steroid production, lifestyle and environment (3-5).

A number of studies have investigated the pathogenesis of osteoporosis at the molecular levels. Two cytokines, including osteoprotegerin and receptor activator of nuclear factor $\kappa \mathrm{B}$ ligand, have been identified as important regulators in the development of osteoporosis $(2,6)$. Members of the Wnt signaling pathway, such as low-density lipoprotein receptor-related protein 5 (LRP5), Wnt3a, secreted Frizzled-related protein 1 and sclerostin (SOST), have been reported to be associated with variation in bone mineral density (7). Additionally, Wnt signaling may enhance osteoblast survival, and interact with parathyroid hormone signaling and bone morphogenetic protein 2, leading to an elevation in osteoblastogenesis (8-10). The transcription factor, specificity protein 1 (Sp1), is associated with a reduction in bone quality and the biomechanical properties of bone (11). However, the underlying etiology of osteoporosis is not yet comprehensively understood and the identification of novel therapeutic targets for osteoporosis is required.

Mesenchymal stem cells (MSCs) from bone marrow are multipotent cells that are able to differentiate into multiple cell lineages, including osteoblasts, adipocytes, fibroblasts and chondrocytes $(12,13)$. The implantation of MSCs has been shown to be an effective and safe method by which to enhance bone regeneration and repair in animal models for bone regeneration as well as in clinical practice $(14,15)$. Gene-expression microarrays are a powerful tool with high-throughput technology, which may be used to assess the expression patterns of multiple genes simultaneously. Therefore, gene expression microarray analysis of MSCs from patients with osteoporosis, may provide novel insights into the mechanisms underlying the pathogenesis of osteoporosis. 
In the present study, gene expression profiles of MSCs from patients with osteoporosis and controls were downloaded, in order to identify differentially expressed genes (DEGs). The screened DEGs were further analyzed using bioinformatics methods to reveal osteoporosis-specific gene expression patterns. The aim was to provide novel targets for the diagnosis and treatment of osteoporosis.

\section{Materials and methods}

Samples and data preprocessing. The gene expression profile of GSE35958 (16) was downloaded from the National Center of Biotechnology Information Gene Expression Omnibus (GEO, http://www.ncbi.nlm.nih.gov/geo/), including five samples of human MSCs from the femoral heads of elderly patients with osteoporosis and four control bone marrow samples from age-matched non-osteoporotic donors. The platform used was GPL570 [HG-U133_Plus_2] Affymetrix Human Genome U133 Plus 2.0 Array (Affymetrix UK Ltd, High Wycombe, United Kingdom).

The downloaded data in CEL files was preprocessed using the Affy package. Background correction and quartile data normalization were performed using the robust multiarray average algorithm (17). Probes without a corresponding gene symbol were then filtered and the average value of gene symbols with multiple probes was calculated. Finally, the expression profile dataset, including 20,539 genes for the nine samples, was obtained.

Screening DEGs. Student's t-test was used to identify DEGs between the osteoporosis and control samples. The Benjamini-Hochberg (BH) procedure (18) was used to adjust the raw P-values into false discovery rates (FDRs). The DEGs were screened using cut-off criteria of FDR $<0.1$ and $\mid \log _{2} \mathrm{FCl}>1.5$.

Functional and pathway enrichment analysis for DEGs. In order to identify biological functions associated with the pathogenesis of osteoporosis, Gene Ontology (GO) (19) functional and Kyoto Encyclopedia of Genes and Genomes (KEGG) (20) pathway enrichment analyses were performed for the identified DEGs, using the online tool of Database for Annotation, Visualization and Integrated Discovery (DAVID) (21) based on the method of Expression Analysis Systemic Explorer (EASE) test (22). The enrichment threshold was an EASE score of 0.1.

Construction of the protein-protein interaction network. Following the acquisition of pathways in which the DEGs with FDR $<0.1$ and $\log _{2} \mathrm{FCl}>1.5$ were markedly enriched, a protein-protein interaction (PPI) network of the significant pathways was constructed, based on the Human Protein Reference Database (HPRD) (23). DEGs, which may convey effective information regarding the pathogenesis of osteoporosis in the constructed PPI network were identified.

Construction of co-change network for pathways. The co-change network for pathways was established based on the method of cumulative hypergeometric probability distribution (24). The pathway-pathway interactions with $\mathrm{P}<0.01$ were identified to construct the co-change network for pathways. $\mathrm{P}$-values were calculated using the following formula:

$$
p=1-\sum_{k=1}^{m-1} \frac{\left(\begin{array}{c}
M \\
k
\end{array}\right)\left(\begin{array}{c}
N-M \\
n-k
\end{array}\right)}{\left(\begin{array}{c}
N \\
n
\end{array}\right)}
$$

Where $\mathrm{N}$ is the total number of protein-protein interactions involved with DEGs, $\mathrm{M}$ is the number of protein-protein interactions associated with DEGs in a pathway, $\mathrm{n}$ is the number of protein-protein interactions involved with DEGs in other pathways and $\mathrm{k}$ is the number of protein-protein interactions involved with DEGs between the two pathways.

Establishment of transcriptional regulatory network for DEGs. TRANSFAC (25) is a database containing information on eukaryotic transcription regulating DNA sequence elements, their genomic binding sites and their DNA-binding profiles. The transcriptional regulatory network for DEGs was constructed based on the TRANSFAC database.

\section{Results}

Identification of differentially expressed genes. Student's t-test and the BH procedure were used to identify DEGs between the osteoporosis and control samples. A total of 1,127 DEGs were identified, with the cut-off criteria of FDR $<0.1$ and $\mid \log _{2} \mathrm{FCl}>1.5$, including 554 upregulated and 573 downregulated DEGs.

Functional and pathway enrichment analysis for DEGs. The screened DEGs were used for functional and pathway enrichment analysis by DAVID. A total of 27 DEGs had significant involvement in the hsa04510 pathway (focal adhesion; FDR=0.0205) and 17 DEGs were significantly enriched in the hsa04142 pathway (lysosome; FDR=0.0477).

Protein-protein interaction network construction. The protein-protein interactions for DEGs involved in the two significant pathways, including focal adhesion and lysosome, were identified, and were used to construct a PPI network (Fig. 1). Table I shows DEGs with a degree $>60$ in the constructed PPI network, including $\beta$-catenin (CTNNB1, 135), SHC-transforming protein 1 (SHC1, 117), RAC- $\alpha$ serine/threonine-protein kinase (AKT1, 117), caveolin 1 (CAV1, 73) and filamin A (FLNA, 63). CTNNB1 and CAV1 were significantly downregulated in the samples from the patients with osteoporosis, while the other three genes were upregulated (Table I).

Establishment of a co-change network for pathways. A total of 227 pathways were annotated for the PPI network and the pathway-pathway interactions with $\mathrm{P}<0.01$ were identified in order to construct the co-change network for pathways (Fig. 2). The co-change pathways with degree $\geq 4$ are shown in Table II. The pathway with the degree of 22 in the constructed co-change network was neuroactive ligand receptor interaction (Table II). Other pathways were inositol phosphate metabolism (degree, 9), cytokine receptor interaction (degree, 5), hematopoietic cell lineage (degree, 5), the calcium signaling pathway (degree, 4) and the chemokine signaling pathway (degree, 4). 
Table I. Five differentially expressed genes with degrees $>60$ in the constructed protein-protein interaction network.

\begin{tabular}{lrrr}
\hline Symbol & Gene ID & Degree & Type \\
\hline CTNNB1 & 1499 & 135 & Downregulated \\
SHC1 & 6464 & 117 & Upregulated \\
AKT1 & 207 & 117 & Upregulated \\
CAV1 & 857 & 73 & Downregulated \\
FLNA & 2316 & 63 & Upregulated \\
\hline
\end{tabular}

CTNNB1, $\beta$-catenin; SHC1, SHC-transforming protein 1; AKT1, RAC- $\alpha$ serine/threonine-protein kinase; CAV1, caveolin 1; FLNA, Filamin A.

Table II. Ten pathways with degrees $\geq 4$ in the constructed co-change network for pathways.

Pathway

Degree

Neuroactive ligand receptor interaction

Inositol phosphate metabolism

Nonhomologous end joining

Cytokine receptor interaction

Hematopoietic cell lineage

Morphine addiction

Maturity onset diabetes of the young

Base excision repair

Calcium signaling pathway

Chemokine signaling pathway

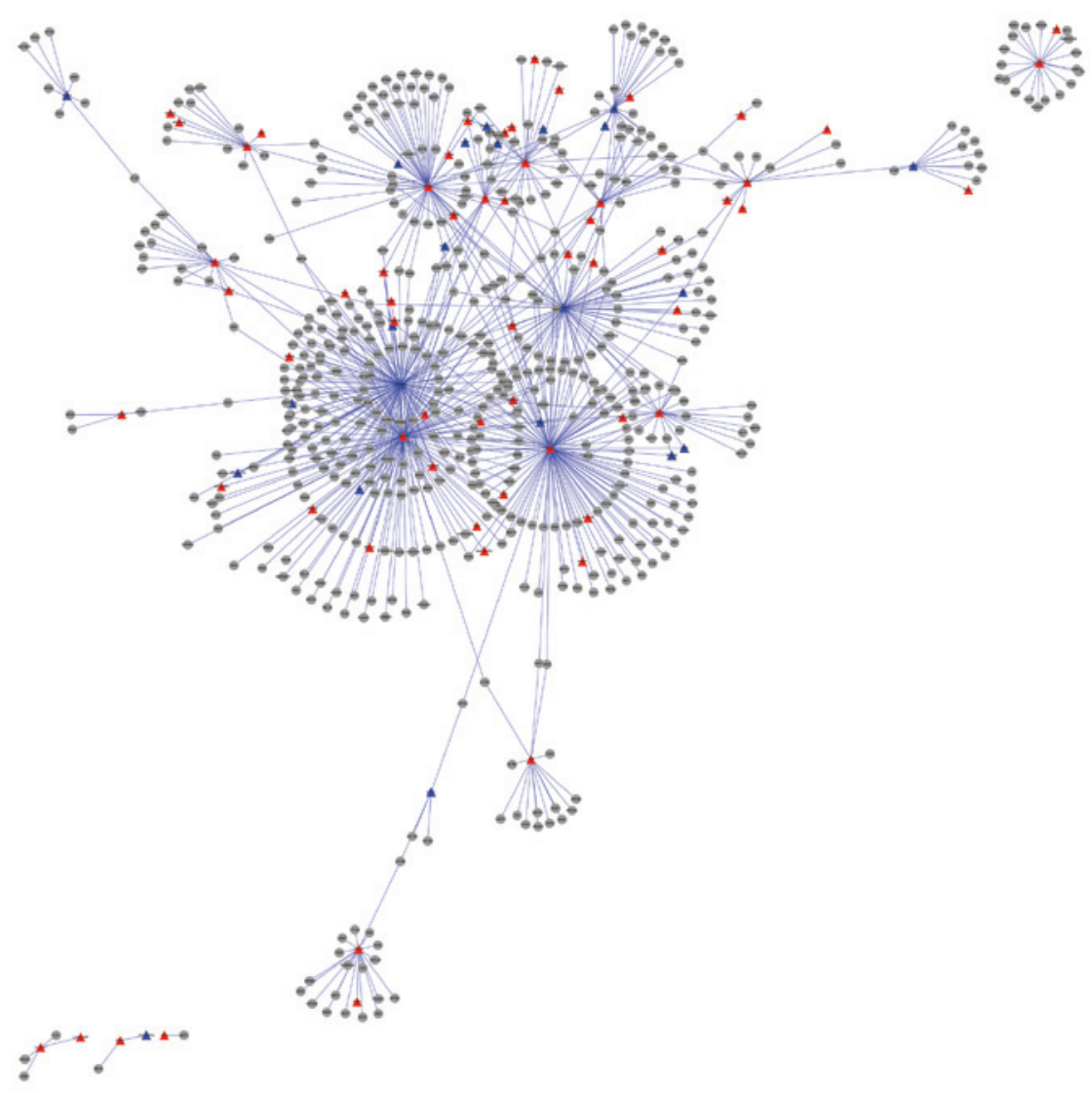

Figure 1. Protein-protein interaction network for DEGs involved in significantly enriched pathways. The red, blue and grey nodes, and grey edges represent the upregulated DEGs, downregulated DEGs, other genes and protein-protein interactions, respectively. DEGs, differentially expressed genes. 
Table III. Nine genes with degrees $\geq 9$ in the constructed transcriptional regulatory network.

\begin{tabular}{lcc}
\hline Symbol & Gene ID & Degree \\
\hline RELA & 5970 & 29 \\
USF1 & 7391 & 27 \\
SP1 & 6667 & 19 \\
FOSL1 & 8061 & 18 \\
CDKN1A & 1026 & 17 \\
USF2 & 7392 & 13 \\
ELK1 & 2002 & 11 \\
JUND & 3727 & 11 \\
RARA & 5914 & 9 \\
\hline
\end{tabular}

RELA, REL-associated protein; USF1, upstream stimulatory factor 1; Sp1, specificity protein 1; FOSL1, FOS-related antigen 1; CDKN1A, cyclin-dependent kinase inhibitor 1A; USF2, upstream stimulatory factor 2; ELK1, ETS domain-containing protein Elk-1; RARA, retinoic receptor $\alpha$.

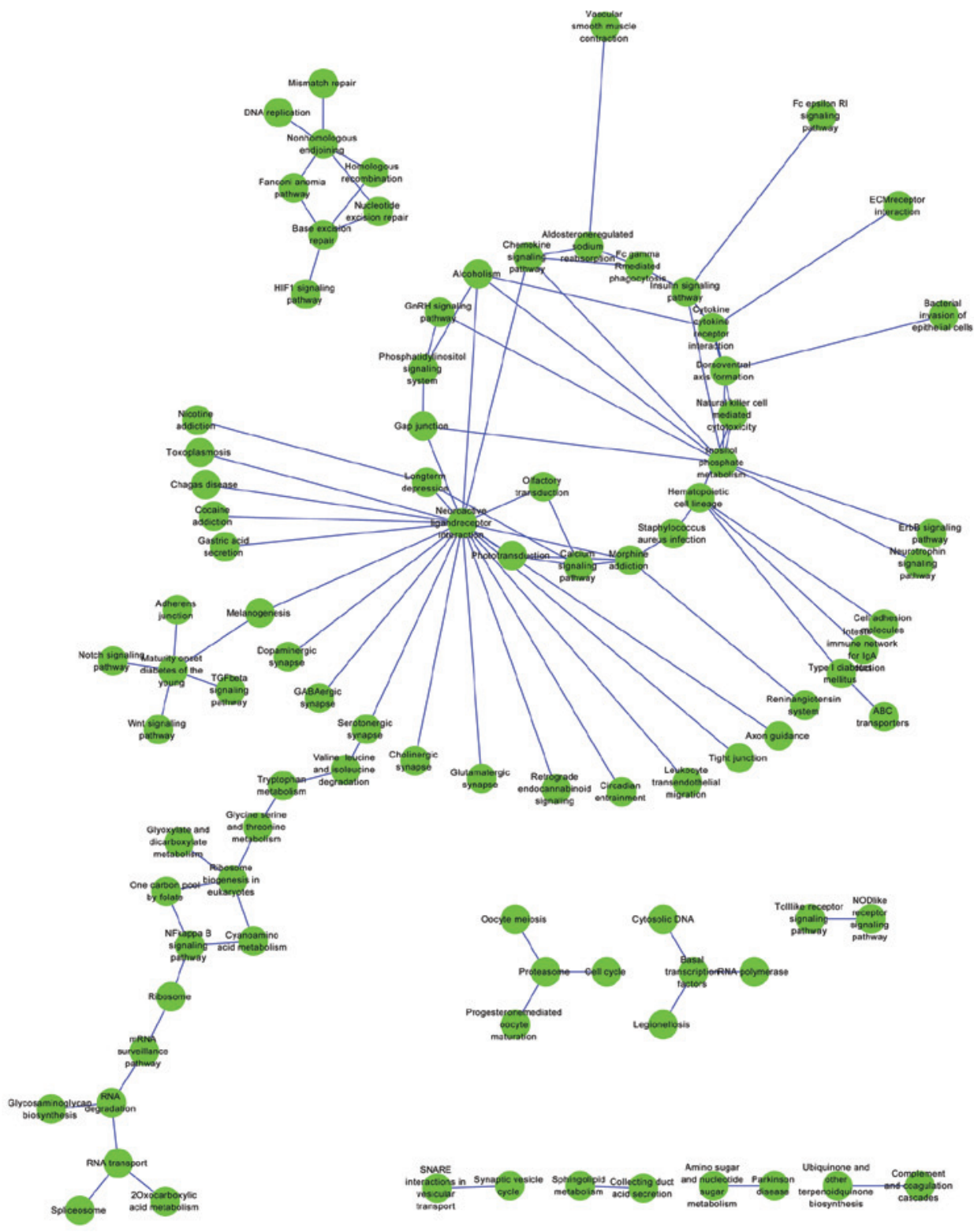

Figure 2. Co-change network for pathways in osteoporosis. The nodes and edges represent the pathways and pathway-pathway interactions, respectively. 

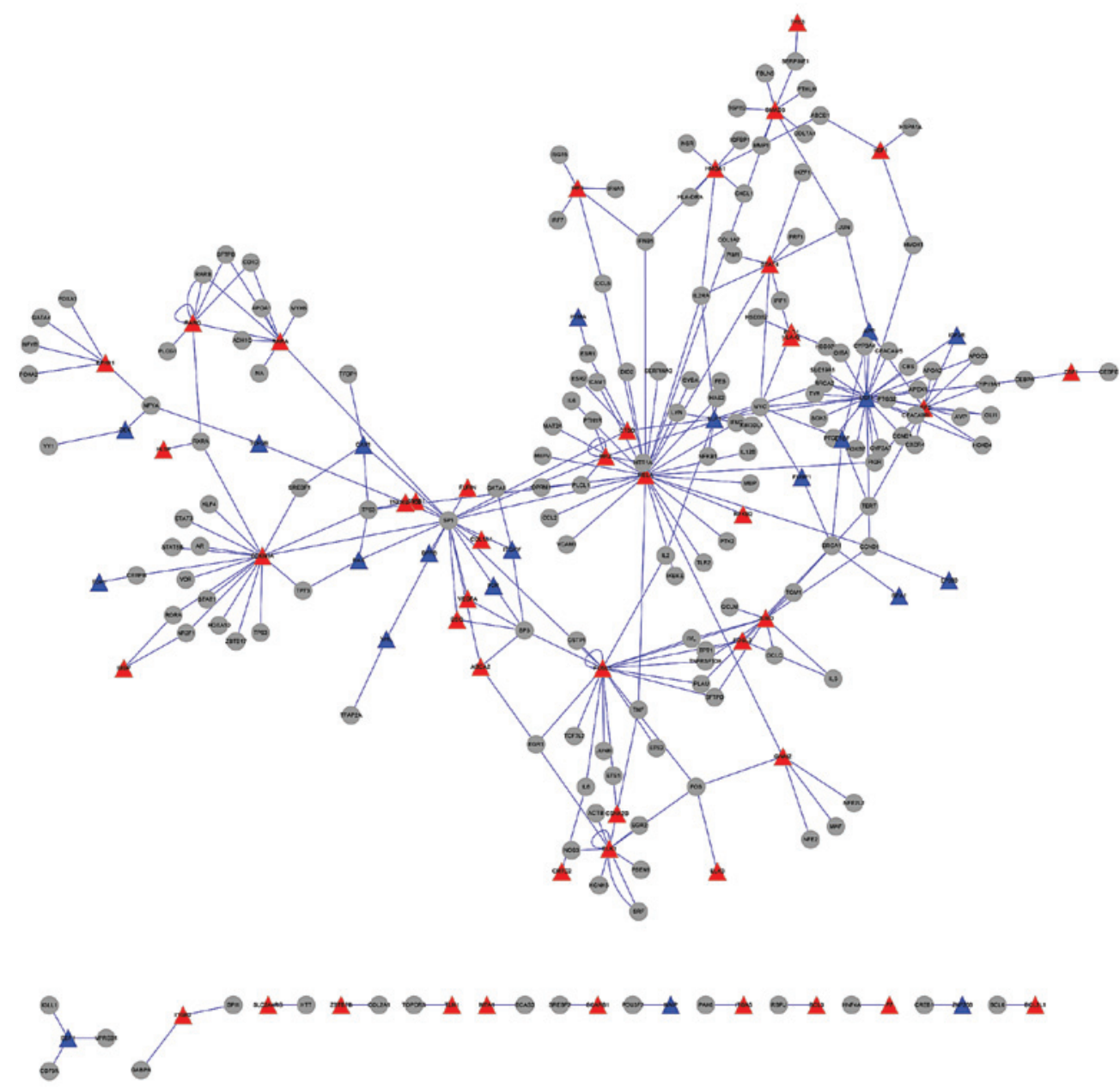

Figure 3. Transcriptional regulatory network for DEGs. The red, blue and grey nodes, and grey edges represent the upregulated DEGs, downregulated DEGs, other genes and transcriptional regulation associations, respectively. DEGs, differentially expressed genes.

Construction of a transcriptional regulatory network for DEGs. A transcriptional regulatory network for DEGs was constructed according to the information included in the TRANSFAC database (Fig. 3). Genes with degrees $\geq 9$ in the transcriptional regulatory network are listed in Table III. The nine genes with high degrees in the constructed transcriptional regulatory network were REL-associated protein (RELA), upstream stimulatory factor 1 (USF1), Sp1, Fos-related antigen 1 (FOSL1), cyclin-dependent kinase inhibitor 1A (CDKN1A), upstream stimulatory factor 2 (USF2), ETS domain-containing protein Elk-1 (ELK1), JUND and retinoic acid receptor $\alpha$ (RARA), with degrees of 29, 27, 19, 18, 17, 13, 11, 11 and 9, respectively (Table III). From the transcriptional regulatory network, Sp1 was shown to have transcriptional regulatory associations with FOSL1, RELA and CDKN1A.

\section{Discussion}

The polymorphisms of a number of genes, including vitamin D receptor, estrogen receptor $\alpha$, estrogen receptor $\beta$, LRP5 and SOST, are associated with a risk of developing osteoporosis $(26,27)$. In the present study, the gene expression profiles of hMSCs samples from elderly patients suffering from osteoporosis and control samples were downloaded. A total of 1,127 DEGs, including 554 upregulated and 573 downregulated DEGs, were screened. Functional and pathway enrichment analyses revealed a significant involvement of DEGs in the pathways of focal adhesion and lysosome. Focal adhesion kinase may regulate the realignment of hMSCs, which is induced by mechanical stretch (28). A number of genes in the focal adhesion family have been reported as candidate genes for osteoporosis (29). Therefore, the signaling pathway of focal adhesion is likely to be important in the pathogenesis of osteoporosis.

The DEGs that were involved in the two significantly enriched pathways were used to construct a PPI network. The gene with degree of 135 in the constructed protein-protein interaction network was CTNNB1. Wnt/ $\beta$-catenin signaling is involved in the anabolic response to mechanical stimulation, and bone mass accrual and maintenance. In addition, $\beta$-catenin has been shown to regulate osteoblast survival and differentiation $(10,30,31)$. Ablation of $\beta$-catenin may promote the differentiation of osteoclast precursors into bone-resorbing osteoclasts, ultimately leading to osteoporosis (32). The results of the current study also showed that CTNNB1 and CAV1 were significantly downregulated in the osteoporosis samples, while SHC1, AKT1 and FLNA were upregulated. 
The pathway with the degree of 22 in the constructed co-change network was neuroactive ligand receptor interaction. The inositol phosphate metabolism, cytokine receptor interaction, hematopoietic cell lineage, calcium signaling and chemokine signaling pathways also had relatively high numbers of interactions. It has been reported that mechanical loading may lead to an increase in the intracellular calcium concentration in osteoblasts, resulting in the activation of AKT, which is responsible for osteoblast survival and proliferation (33). Certain chemokines are essential for bone metabolism, such as osteopontin, which has been reported to be involved in the pathogenesis of osteoporosis (34). Therefore, the pathway of neuroactive ligand receptor interaction may be important in the development of osteoporosis.

The genes with high degrees in the constructed transcriptional regulatory network, were RELA, USF1, SP1, FOSL1, CDKN1A, USF2, ELK1, JUND and RARA. Activation of liver $\mathrm{X}$ receptor upregulates the expression of osteoclast/macrophage-related markers, including USF1/2, which has the potential to inhibit the differentiation of bone marrow-derived osteoclast precursors into osteoclasts (35). Furthermore, Sp1 had associations with the transcriptional regulation of FOSL1, RELA and CDKN1A. The collagen type I $\alpha 1$ and Sp1 polymorphisms are associated with reduced bone density and osteoporosis (36). Overexpression of Fos-related antigen 1, encoded by FOSL1, may increase bone formation and accelerate osteoblast differentiation in mice (37). Therefore, FOSL1, RELA and CDKN1A may also be also involved in the pathogenesis of osteoporosis.

In conclusion, the significant DEGs identified in the constructed PPI network and transcriptional regulatory network may provide useful information on the pathogenesis of osteoporosis. However, the present study did not analyze hMSCs from patients of different ages and genders. Furthermore, the results of the study require confirmation by experimental research. Therefore, the molecular mechanism underlying the development of osteoporosis demand further exploration.

\section{Acknowledgements}

This study was funded by Key Disciplines Group Construction Project of Pudong Health Bureau of Shanghai (PWZxq2014-9) and Shanghai Municipal Commission of Health and Family Planning (grant no. 201440050).

\section{References}

1. Nguyen TV and Eisman JA: Genetic profiling and individualized assessment of fracture risk. Nat Rev Endocrinol 9: 153-161, 2013.

2. Rachner TD, Khosla S and Hofbauer LC: Osteoporosis: now and the future. Lancet 377: 1276-1287, 2011.

3. Ralston SH and Uitterlinden AG: Genetics of osteoporosis. Endocr Rev 31: 629-662, 2010.

4. Pietschmann P, Rauner M, Sipos W and Kerschan-Schindl K: Osteoporosis: an age-related and gender-specific disease - a mini-review. Gerontology 55: 3-12, 2009.

5. Seeman E: Bone quality: the material and structural basis of bone strength. J Bone Miner Metab 26: 1-8, 2008.

6. Kong YY, Yoshida H, Sarosi I, et al: OPGL is a key regulator of osteoclastogenesis, lymphocyte development and lymph-node organogenesis. Nature 397: 315-323, 1999.

7. Sims AM, Shephard N, Carter K, et al: Genetic analyses in a sample of individuals with high or low BMD shows association with multiple Wnt pathway genes. J Bone Miner Res 23: 499-506, 2008.
8. Kramer I, Keller H, Leupin O and Kneissel M: Does osteocytic SOST suppression mediate PTH bone anabolism? Trends Endocrinol Metab 21: 237-244, 2010.

9. Rawadi G, Vayssière B, Dunn F, Baron R and Roman-Roman S: BMP-2 controls alkaline phosphatase expression and osteoblast mineralization by a Wnt autocrine loop. J Bone Miner Res 18: 1842-1853, 2003.

10. Almeida M, Han L, Bellido T, Manolagas SC and Kousteni S: Wnt proteins prevent apoptosis of both uncommitted osteoblast progenitors and differentiated osteoblasts by beta-catenin-dependent and -independent signaling cascades involving Src/ERK and phosphatidylinositol 3-kinase/AKT. J Biol Chem 280: 41342-41351, 2005.

11. Jin H, Stewart TL, Hof RV, Reid DM, Aspden RM and Ralston S: A rare haplotype in the upstream regulatory region of COL1A1 is associated with reduced bone quality and hip fracture. $\mathrm{J}$ Bone Miner Res 24: 448-454, 2009.

12. Kilian KA, Bugarija B, Lahn BT and Mrksich M: Geometric cues for directing the differentiation of mesenchymal stem cells. Proc Natl Acad Sci USA 107: 4872-4877, 2010.

13. Valtieri $M$ and Sorrentino A: The mesenchymal stromal cell contribution to homeostasis. J Cell Physiol 217: 296-300, 2008.

14. Griffin M, Iqbal SA and Bayat A: Exploring the application of mesenchymal stem cells in bone repair and regeneration. J Bone Joint Surg Br 93: 427-434, 2011.

15. Jones E and Yang X: Mesenchymal stem cells and bone regeneration: current status. Injury 42: 562-568, 2011.

16. Benisch P, Schilling T, Klein-Hitpass L, et al: The transcriptional profile of mesenchymal stem cell populations in primary osteoporosis is distinct and shows overexpression of osteogenic inhibitors. PLoS One 7: e45142, 2012.

17. Irizarry RA, Bolstad BM, Collin F, Cope LM, Hobbs B and Speed TP: Summaries of affymetrix GeneChip probe level data. Nucleic Acids Res 31: e15, 2003.

18. Benjamini Y and Hochberg Y: Controlling the false discovery rate: a practical and powerful approach to multiple testing. J Roy Statist Soc Ser B Stat (Methodological) 57: 289-300, 1995.

19. Ashburner, M., et al., Gene Ontology: tool for the unification of biology. Nature genetics, 2000. 25(1): p. 25-29

20. Kanehisa, M. and S. Goto, KEGG: kyoto encyclopedia of genes and genomes. Nucleic acids research, 2000. 28(1): p. 27-30

21. Da Wei Huang, B.T.S. and R.A. Lempicki, Systematic and integrative analysis of large gene lists using DAVID bioinformatics resources. Nature protocols, 2008. 4(1): p. 44-57

22. Hosack DA, Dennis G Jr, Sherman BT, Lane HC and Lempicki RA: Identifying biological themes within lists of genes with EASE. Genome Biol 4: R70, 2003.

23. Stelzl U, Worm U, Lalowski M, et al: A human protein-protein interaction network: a resource for annotating the proteome. Cell 122: 957-968, 2005.

24. Przulj N, Wigle DA and Jurisica I: Functional topology in a network of protein interactions. Bioinformatics 20: 340-348, 2004.

25. Wingender E, Dietze P, Karas H and Knüppel R: TRANSFAC: a database on transcription factors and their DNA binding sites. Nucleic Acids Res 24: 238-241, 1996.

26. Li WF, Hou SX, Yu B, Li MM, Férec C and Chen JM: Genetics of osteoporosis: accelerating pace in gene identification and validation. Hum Genet 127: 249-285, 2010.

27. Jin H and Ralston SH: Regulatory polymorphisms and osteoporosis. In Gene Regulatory Sequences and Human Disease. Springer, New York, NY, p41-p54, 2012.

28. Xu B, Song G and Ju Y: Effect of focal adhesion kinase on the regulation of realignment and tenogenic differentiation of human mesenchymal stem cells by mechanical stretch. Connect Tissue Res 52: 373-379, 2011.

29. Zintzaras E, Doxani C, Koufakis T, Kastanis A, Rodopoulou P and Karachalios T: Synopsis and meta-analysis of genetic association studies in osteoporosis for the focal adhesion family genes: the CUMAGAS-OSTEOporosis information system. BMC Med 9: 9, 2011.

30. Westendorf JJ, Kahler RA and Schroeder TM: Wnt signaling in osteoblasts and bone diseases. Gene 341: 19-39, 2004.

31. Lau KH, Kapur S, Kesavan C and Baylink DJ: Up-regulation of the Wnt, estrogen receptor, insulin-like growth factor-I, and bone morphogenetic protein pathways in C57BL/6 J osteoblasts as opposed to $\mathrm{C} 3 \mathrm{H} / \mathrm{HeJ}$ osteoblasts in part contributes to the differential anabolic response to fluid shear. J Biol Chem 281: 9576-9588, 2006. 
32. Otero K, Shinohara M, Zhao H, et al: TREM2 and $\beta$-catenin regulate bone homeostasis by controlling the rate of osteoclastogenesis. J Immunol 188: 2612-2621, 2012.

33. Rangaswami H, Schwappacher R, Tran T, et al: Protein kinase G and focal adhesion kinase converge on Src/Akt/ $\beta$-catenin signaling module in osteoblast mechanotransduction. J Biol Chem 287: 21509-21519, 2012.

34. Altintas A, Saruhan-Direskeneli G, Benbir G, Demir M and Purisa S: The role of osteopontin: a shared pathway in the pathogenesis of multiple sclerosis and osteoporosis? J Neurol Sci 276: 41-44, 2009.

35. Robertson Remen KM, Gustafsson JA and Andersson G: The liver $\mathrm{X}$ receptor promotes macrophage differentiation and suppresses osteoclast formation in mouse RAW264.7 promyelocytic leukemia cells exposed to bacterial lipopolysaccharide. Biochem Biophys Res Commun 430: 375-380, 2013.
36. Grant SF, Reid DM, Blake G, Herd R, Fogelman I and Ralston SH: Reduced bone density and osteoporosis associated with a polymorphic Sp1 binding site in the collagen type I alpha 1 gene. Nat Genet 14: 203-205, 1996.

37. Jochum W, David JP, Elliott C, et al: Increased bone formation and osteosclerosis in mice overexpressing the transcription factor Fra-1. Nat Med 6: 980-984, 2000. 